\title{
Modulating TSH Receptor Signaling for Therapeutic Benefit
}

\author{
Gerd Krause $^{a}$ Anja Eckstein ${ }^{c}$ Ralf Schülein ${ }^{b}$

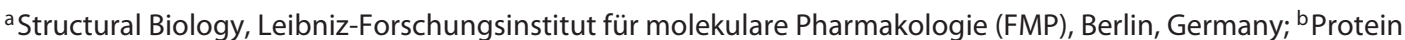 \\ Trafficking, Leibniz-Forschungsinstitut für molekulare Pharmakologie (FMP), Berlin, Germany; ${ }^{C}$ Department of \\ Ophthalmology, University Hospital Essen, Essen, Germany
}

\section{Keywords}

Insulin-like growth factor 1 receptor · Thyroid-stimulating hormone receptor - Crosstalk · Graves' orbitopathy · Graves' disease

\begin{abstract}
Autoimmune thyroid-stimulating antibodies are activating the thyrotropin receptor (TSHR) in both the thyroid and the eye, but different molecular mechanisms are induced in both organs, leading to Graves' disease (GD) and Graves' orbitopathy (GO), respectively. Therapy with anti-thyroid drugs to reduce hyperthyroidism (GD) by suppressing the biosynthesis of thyroid hormones has only an indirect effect on GO, since it does not causally address pathogenic TSHR activation itself. GO is thus very difficult to treat. The activated TSHR but also the cross-interacting insulin-like growth factor 1 receptor (IGF-1R) contribute to this issue. The TSHR is a heptahelical G-protein-coupled receptor, whereas the IGF-1R is a receptor tyrosine kinase. Despite these fundamental structural differences, both receptors are phosphorylated by $\mathrm{G}$-protein receptor kinases, which enables $\beta$-arrestin binding. Arrestins mediate receptor internalization and also activate the mitogen-activated protein kinase pathway.
\end{abstract}

Moreover, emerging results suggest that arrestin plays a critical role in the cross-interaction of the TSHR and the IGF-1R either in their common signaling pathway and/or during an indirect or potential TSHR/IGF-1R interaction. In this review, novel pharmacological strategies with allosteric small-molecule modulators to treat GO and GD on the level of the TSHR and/or the TSHR/IGF-1R cross-interaction will be discussed. Moreover, monoclonal antibody approaches targeting the TSHR or the IGF-1R and thereby preventing activation of either receptor will be presented. Another chapter addresses the immunomodulation to treat GO using TSHR-derived peptides targeting the human leukocyte antigen DR isotope (HLA-DR), which is a feasible approach to tackle GO, since HLA-DR and TSHR are overexpressed in orbital tissues of GO patients.

(C) 2020 European Thyroid Association Published by S. Karger AG, Basel

\section{Introduction}

\section{TSH-Receptor, Graves' Disease and Graves'}

Orbitopathy

The thyrotropin receptor or thyroid-stimulating hormone (TSH) receptor (TSHR) is mainly expressed in fol- 
licular epithelial cells of the thyroid gland [1]. TSHR activation by TSH leads to stimulation of secondary messenger pathways involving predominantly cAMP but also inositol 1,4,5-trisphosphate $\left(\mathrm{IP}_{3}\right)[2,3]$. Activation of the ERK pathway by TSHR via $\beta$-arrestin 1 is involved in osteoblast homeostasis [4].

Pathological activation of the TSHR by TSHR-stimulating autoimmune antibodies (TSAb) leads to the uncontrolled production of thyroid hormones $\mathrm{T} 3$ and $\mathrm{T} 4 \mathrm{in}$ the thyroid causing hyperthyroidism in Graves' disease (GD) [5]. The TSAb binds to the TSHR at a similar extracellular site as TSH itself and causes deregulated TSHR hyperactivation [6]. To date, antithyroid drugs are used to reduce hyperthyroidism by correcting the "clinically evident" consequences. Here, the activity of the enzyme thyroid peroxidase is inhibited, thereby impairing thyroid hormone synthesis and release. However, pathological activation of the TSHR by TSAbs is not causally addressed. To date, there is no therapy available targeting the activated TSHR itself. Therefore, if the pathological antibody production does not calm down during the course of antithyroid drug treatment (remission), the thyroid will be removed either by radioiodine therapy or by surgical thyroidectomy and, as a consequence, L-thyroxin has to be substituted lifelong in these patients (summary in [7]).

In addition to follicular epithelial cells of the thyroid gland and osteoblasts, the TSHR is also expressed in retro-orbital fibroblasts [1]. Thus, autoimmune TSAbs also activate the TSHR in the orbit causing Graves' orbitopathy (GO), a quality of life-reducing, appearance-changing, and sight-threatening disease. It is characterized by inflammatory symptoms, extraocular muscle fibrosis, and adipose tissue growth leading to protrusion of the eyes, diplopia, and blurred vision. About half of GD patients develop GO and 3-5\% develop a severe sightthreatening course of disease [8].

Of note, while autoimmune TSAbs are activating the TSHR in both the thyroid and orbit, different molecular mechanisms are activated in the orbit. The therapy using antithyroid drugs decreasing thyroid hormone synthesis does not target the TSHR itself and has, thus, only an indirect and very limited effect on GO (poor control of thyroid function is a risk for more severe course of GO). The autoimmune reaction in the orbit leads finally to the infiltration of orbital tissues with predominantly macrophages and $\mathrm{T}$ cells and to the release of several potent mediators of inflammation. Therefore, today's primary therapy for GO are anti-inflammatory immunosuppressives. Steroids are the first-line therapy, followed by im- munosuppressive drugs if the therapy effect is not or not sufficiently achieved. A careful look at the outcome measures in all treatment studies reveals that all these drugs are potent in inflammatory reductions [9]. However, the drugs are only partially, if at all, effective to improve a pathologically reduced mobility of the eye muscles or to reduce a once developed exophthalmus (CIRTED, Mycophenolatmofetil [MMF], Tocilizumab study, Rituximab $[10,11])$.

Orbital fibroblasts (OF) have been recognized as primary target cells of TSAb, and TSHR acts as a primary autoantigen also in GO [12]. In OFs, direct activation of the TSHR by TSAb leads to production of extracellular matrix, including hyaluronan (HA). TSAb ligation additionally induces a pathologic crosstalk between the TSHR and the insulin-like growth factor 1 receptor (IGF-1R), which increases HA production [13]. This finally causes swelling and disruption of the extraocular muscles, which finally leads to fibrosis [12]. TSHR stimulation does also lead to adipogenic differentiation of OFs. There is considerable evidence that expression of both TSHR and the IGF-1R in the OF and orbital adipocytes contributes to the difficulty to treat GO $[14,15]$. The IGF-1R contributes also to HA production in GO. The elucidation of these pathological signaling mechanisms opened the door for direct targeting therapy for GO. Two randomized clinical trials (one of which was an FDA approval study) showed that targeting the IGF-1R with blocking antibodies led to a significant reduction of proptosis (median $3 \mathrm{~mm}$ ) and improvement of eye motility in addition to inflammation reduction [16-18]. It is still not clear if teprotumumab is only effective in the early phase of the disease or also later in the course.

In summary, there is no targeted therapy available for Graves' hyperthyroidism, while the pharmacological treatment of GO is already promising with targeting the IGF-1R; however, it is still unclear whether the development of optic nerve compression can be prevented or treated with this new drug. In addition, we need to find out if directly targeting the TSHR with blocking antibodies or small-molecule antagonists is even more efficient in the treatment of GD and GO.

In this review, new pharmacological approaches and modulators for GO and GD are discussed, which directly target the TSHR. The crosstalk/transactivation of the TSHR and the IGF-1R will also be considered. First, the structural aspects and common and differing molecular activation mechanisms of the two receptors will be addressed. 


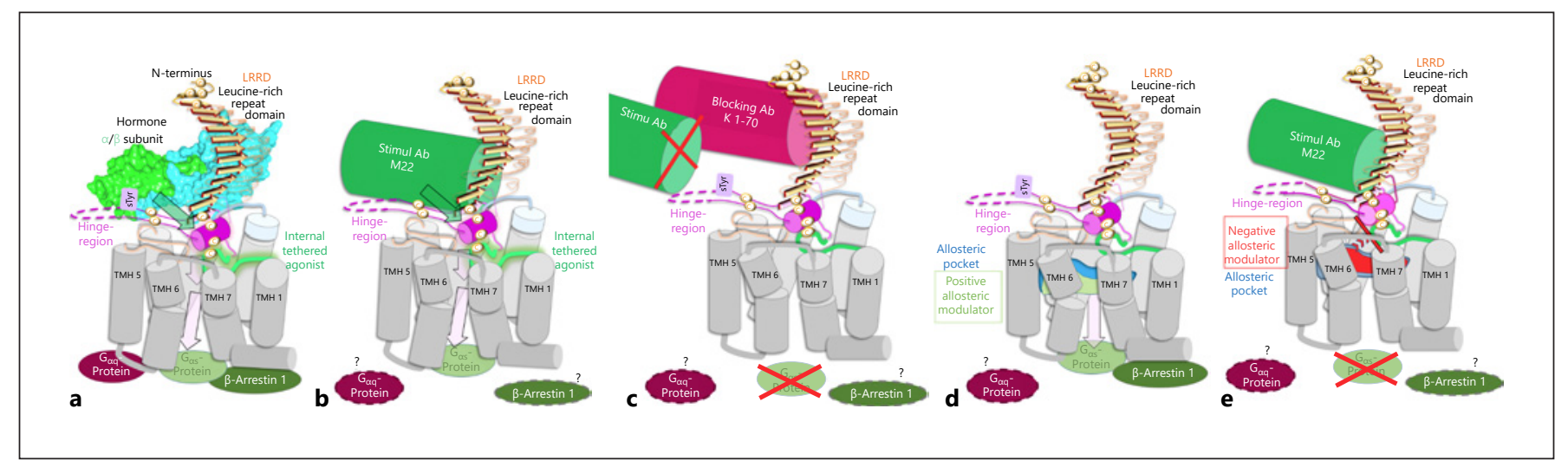

Fig. 1. Schemes of TSHR activation/inhibition. a TSH binds to LRRD (beige) and hinge (magenta) regions. Signaling via an internal agonist (light green; linked via disulfide bonds, yellow) and delocalization of TM conformations (grey) leads to Gas (olive) or Gaq (dark red) coupling or $\beta$-arrestin 1 recruitment (dark green). b Stimulating antibody (Ab) M22 (green) binds like TSH and activates Gas. Effects on other pathways are unknown. c Blocking Ab

\section{TSHR Activation and Inhibition at the Molecular Level}

The molecular structure of the TSHR is characterized by a large extracellular ectodomain (410 amino acids) consisting of a leucine-rich repeat domain (LRRD), a hinge region [19], and an internal sequence, which acts as a tethered agonist followed by a heptahelical transmembrane domain and a C-terminal tail [20] like in other Gprotein-coupled receptors (GPCRs) of family A.

To date, crystal structures have been published only for fragments of the TSHR, such as for the LRRD, with bound activating (M22 [21]) and blocking (K-71 [22]) monoclonal antibodies. For structure function studies, homology models of the entire TSHR were assembled using, on the one hand, the structural fragments of the ectodomains of the TSHR and the homologous folliclestimulating hormone receptor (FSHR) including parts of the hinge region as template [23]. On the other hand, a model of the whole transmembrane domain was built using the respective crystal structures of homologous GPCRs $[20,24]$.

TSH (like the TSAb) binds exclusively to the large extracellular region of the TSHR (Fig. 1a, b) [25]. Amino acid residues involved in TSH binding, like those of the LRRD and the sulfated tyrosine (sTyr) [26] in the hinge region (magenta in Fig. 1), have been described in detail [27]. TSH and TSAb binding leads to structural alteration of various extracellular parts. These changes finally con-
K1-70 (red) inhibits TSAb function. Other effects of this antibody are unknown. d PAM (pale green) binds in an allosteric pocket (blue), activates Gas and recruits (biased) $\beta$-arrestin 1 depending on the ligand. e NAM (red) impairs Gas activation induced by TSH or TSAb; other pathways are unknown. PAM, positive allosteric modulator; NAM, negative allosteric modulator.

verge in a conformation of the TSHR sequence (405 FNCPCEDIMGY 414), known as "intramolecular agonist" or "tethered ligand" (light green in Fig. 1, 4). It is embedded between the extracellular loops [28] and engenders an active state of the TSHR, which in turn induces multiple changes in the transmembrane helix (TMH) bundle [29]. This finally enables binding and activation of effectors such as the G-proteins Gas and Gaq or $\beta$-arrestins at the intracellular side of the receptor [30] (Fig. 1). Very recently, it was shown that also Gai/o couples to the TSHR and plays a role in biphasic cAMP formation [31].

TSHR-stimulating monoclonal antibodies, like M22 [21], bind to the orthosteric binding site of TSH on the extracellular LRR domain [32] and activate Gas (Fig. 1b). TSHR antibodies with antagonistic effects like K1-70 [22] bind somewhat shifted to the N-terminal end of the extracellular LRR domain and, thus, block the TSHR activation induced by TSH or TSAb (Fig. 1c). It is conceivable that many of the small-molecule ligands of the TSHR can bind allosterically as agonists (positive allosteric modulator, pale green in Fig. 1d) or as antagonists (negative allosteric modulator, red in Fig. 1e, see also smallmolecule antagonists in Table 1). The binding pocket for these small molecules (blue in Fig. 1d, e) within the transmembrane helices of the TSHR correlates in many cases with the orthosteric binding site of the GPCRs belonging to the rhodopsin-like GPCR family [33].
Krause/Eckstein/Schülein 
Table 1. Summary of potential GO and GD modulators, which were previously described or are currently under development

\begin{tabular}{|c|c|c|c|c|c|c|}
\hline Type & Inhibitor & Ref. & Target & Advantages & Limitations & $\begin{array}{l}\text { Potential } \\
\text { therapy }\end{array}$ \\
\hline \multirow[t]{3}{*}{$\begin{array}{l}\text { Monoclonal } \\
\text { antibodies }\end{array}$} & K 1-70 & {$[54]$} & & $\begin{array}{l}\text { Highly receptor specific; } \\
\text { high affinity }\end{array}$ & $\begin{array}{l}\text { High costs production, } \\
\text { storage, transport; }\end{array}$ & \\
\hline & & & LRRD & Clinical trial I (UK) & $\begin{array}{l}\text { IV application, } \\
\text { biased inhibition difficult }\end{array}$ & GO, FTC \\
\hline & Teprotumumab & {$[18]$} & $\begin{array}{l}\text { IGF-1R } \\
\text { CR domain }\end{array}$ & Clinical trial III & $\begin{array}{l}\text { No complete remission } \\
\text { in all patients }\end{array}$ & $\mathrm{GO}$ \\
\hline \multirow[t]{3}{*}{ Peptides } & ATX-GD-59 & {$[58]$} & TSHR & Immunogenic clinical trial I & & GD \\
\hline & TSHR 200-213 & {$[57]$} & LRRD & In vivo disease model & $\begin{array}{l}\text { Digestive degradation } \\
\text { possible }\end{array}$ & $\mathrm{GO}$ \\
\hline & FNPCKDIMGY & {$[28]$} & $\begin{array}{l}\text { E->K modified } \\
\text { internal agonist }\end{array}$ & & $\begin{array}{l}\text { Inhibits TSHR but also } \\
\text { LHR and FSHR }\end{array}$ & \\
\hline \multirow{5}{*}{$\begin{array}{l}\text { Small } \\
\text { molecules, } \\
\text { NAM }\end{array}$} & Antag2 & {$[67]$} & TSHR & Good oral bioavailability, & & \\
\hline & Antag3 & {$[68]$} & $\begin{array}{l}\text { allosteric } \\
\text { binding pocket }\end{array}$ & $\begin{array}{l}\text { low-cost production and } \\
\text { storage }\end{array}$ & Missing high affinity yet & \\
\hline & Org274179 & {$[70]$} & within the & Biased inhibition, adressable & \multirow{3}{*}{$\begin{array}{l}\text { Awaiting application } \\
\text { of disease model yet }\end{array}$} & \multirow{3}{*}{$\mathrm{GD}, \mathrm{GO}$} \\
\hline & VA-K-14 & {$[71]$} & TMD & diminishing side effects & & \\
\hline & S37a & $\begin{array}{l}{[72]} \\
{[73]}\end{array}$ & $\begin{array}{l}\text { New allosteric } \\
\text { site }\end{array}$ & Highly TSHR selective & & \\
\hline
\end{tabular}

Monoclonal antibodies, peptides, and small molecules as negative allosteric modulators (NAM) targeting mainly the TSHR except for teprotumumab that targets the IGF-1R. Advantages and limitations that apply to all members of a type are printed in italics.

\section{IGF-1R Activation at the Molecular Level}

The molecular structure of the IGF-1R is characterized on the extracellular side by a short LRRD (marked as L1 in Fig. 2), a cysteine rich domain (CR), a second short LRRD (L2), and 3 fibronectin type III domains (FnIII-1, -2 , and -3 ), which are followed by a single-helix transmembrane domain. On the intracellular side, the IGF-1R possesses an intracellular tyrosine kinase (TK) domain and its C-terminal tail. A recent cryo-EM structure (PDB id: $6 \mathrm{PYH}$ ) of the extracellular domains in the active state [34] reveals that 2 protomers (dark and pale green in Fig. 2) are linked by multiple disulfide bonds to form a stable covalent asymmetric homodimer. Li et al. [34] provided a working model suggesting that in the autoinhibited inactive state, the 2 FnIII-3 domains are spatially separated by 67 Angstrom preventing the TK domain from autophosphorylation (Fig. 2a). The binding site for one IGF-1 molecule (yellow in Fig. 2b) is formed by the L1 and CR domains of one IGF-1R protomer and by the $\alpha$-CT and FnIII-1 domains of the other protomer. IGF-1 induces conformational changes by which the intracellu- lar TK domains are merged and autophosphorylation of IGF-1R occurs (Fig. 2b).

\section{The Common Role of $\beta$-Arrestin in TSHR and IGF-1R Signaling in GO}

After TSHR activation, $\beta$-arrestins are recruited to the receptor as in most GPCRs by recognizing receptor phosphorylation of the C-terminal tail caused by G-proteincoupled receptor kinases (GRK) [35].

$\beta$-Arrestin has 2 main tasks, initializing receptor desensitization/internalization (clathrin-mediated endocytosis) and/or activation of mitogen-activated protein kinase (MAPK). The latter activation is followed by phosphorylation of extracellular signal-regulated kinases (ERK), p38, and AKT (protein kinase B) $[4,36]$. The TSHR is predominantly internalized by $\beta$-arrestin 2 [37]. TSH-induced $\beta$-arrestin 1 signaling via MAPK/ERK is involved in osteoblast differentiation [4]. Arrestin 1 scaffolds TSHR and IGF-1R to enable crosstalk [37]. 
Fig. 2. The IGF-1R possesses extracellularly 2 short leucine-rich repeat domains (L1, L2), a cysteine-rich domain (CR) and 3 FnIII domains. A single transmembrane domain is followed by an intracellular TK domain. Two IGF-1R protomers (dark and pale green) form a homodimer. Schemes of IGF-1R working models are based on cryoEM structures [34]. a Autoinhibited inactive state with distant TK domains. b IGF-1 (yellow) induces activation and conformational changes that merge the TK domains. This enables trans-autophosphorylation of the TK domain initiating kinase signaling. c G-protein receptor kinases (GRK) phosphorylate serine residues on $\mathrm{C}$-terminal tail enabling $\beta$-arrestin binding and subsequent signaling/internalization.

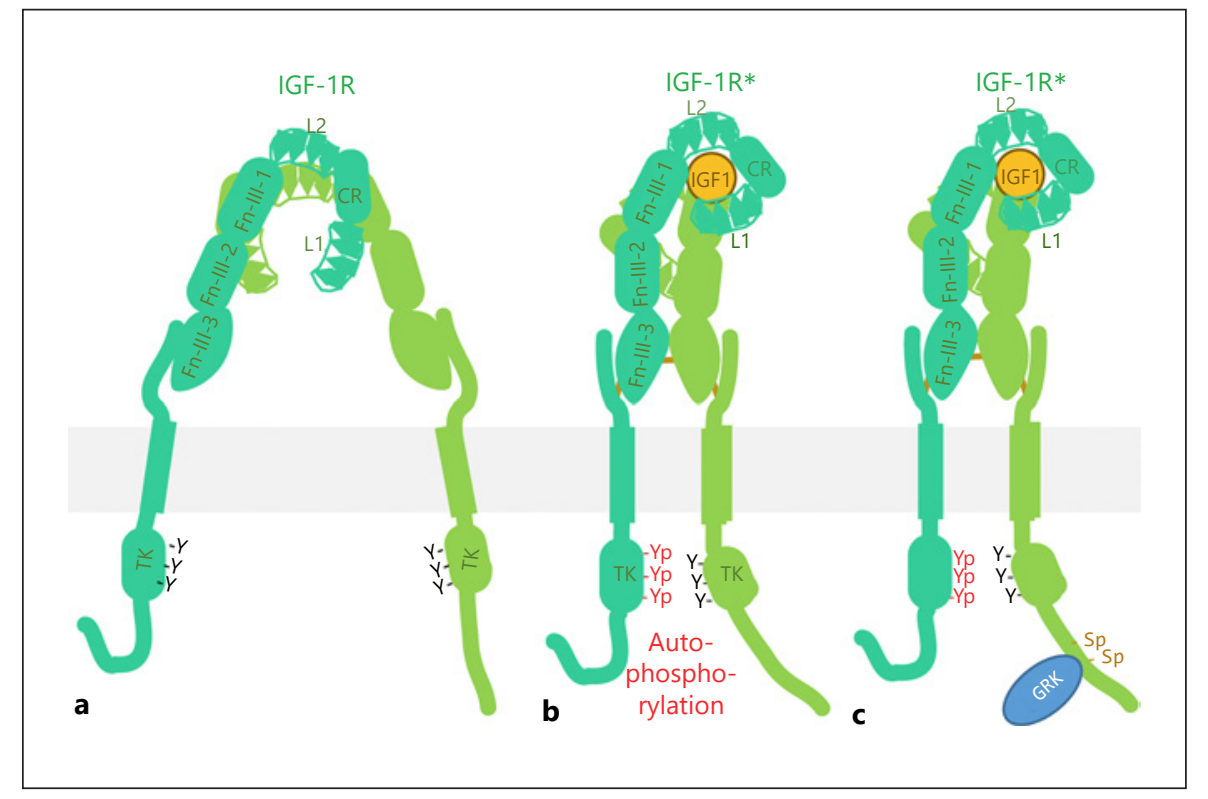

Autophosphorylation of the IGF-1R on tyrosine residues (Y1161, Y1165, Y1166) in response to IGF-1 binding occurs in trans, i.e., one subunit of the dimeric receptor phosphorylates tyrosine residues on the TK domain of the other subunit (Fig. 2b), leading to activation of the 2 main signaling pathways: the PI3K-AKT/PKB pathway and the Ras-MAPK pathway [38].

In addition, there are further signaling mechanisms, since it is thought that the IGF-1R forms a functional hybrid with a GPCR (receptor TK [RTK]/GPCR hybrid) [38]. This integrates canonical TK signaling with noncanonical GPCR signaling [39]. Following G-protein coupling (mainly $\mathrm{Gi}$ ), the IGF-1R is rapidly phosphorylated by GRKs (GRK 2 and 6) at serine residues (S1248p, S1291p; Fig. 2 c) within the C-terminus [40], which are then recognized by $\beta$-arrestin (Fig. 3).

Consequently, IGF-1R also signals through $\beta$-arrestin, which has threefold functions in this case. It is a mediator of IGF-1R downregulation by $\beta$-arrestin-distorted ubiquitination [41], it prevents further G-protein coupling $[42,43]$, and it is as an additional mediator of RTK-independent MAPK signaling.

IGF-1 binding, thus, results in a balanced activation of kinase and G-protein signaling and leads to desensitization and internalization. Unbalanced signaling, or biased signaling of the IGF-1R, can be induced (1) by antibodies or by IGF-1R transactivation (e.g., by a GPCR, which has not been experimentally confirmed yet) leading to $\beta$-arrestin-biased enhancement of IGF-1R ubiquitina- tion. Moreover, the $\beta$-arrestin 1-mediated ERK activation occurs even when the classical IGF-1R kinase signaling is impaired (such as by blocking ligands). On the other hand, biased kinase/G-protein signaling of IGF-1R can occur (2) when $\beta$-arrestin 1 recruitment is impaired and IGF-1R phosphorylation of TK is enhanced [31].

\section{Crosstalk or Transactivation of the TSHR/IGF-1R}

On the molecular level, it is a matter of debate whether TSHR and IGF-1R influence each other via overlapping signaling pathways or whether they interact physically by direct heterodimerization. It might also be possible that both proteins interact via other proteins, such as $\beta$-arrestin, or that GO-Igs are also able to bind and activate IGF-1R. An interaction of both receptors was construed from membrane co-localization and co-immunoprecipitation [44]. These methods are a valuable hint but not a reliable proof of a direct protomer interaction. As an alternative to a direct protomer interaction, the presence of a signalosome has been proposed, in which $\beta$-arrestin 1 mediates proximity and crosstalk of the TSHR and IGF-1R (Fig. 3a). This has been shown by a proximity ligation assay (indicating a distance of max. $\sim 40 \mathrm{~nm}$ ) and $\beta$-arrestin 1 knockdown [45]. Smith and Janssen [16] hypothesized that some GO-Igs are able to bind and activate the IGF-1R. Marcus-Samuels and coworkers $[46,47]$, in contrast, provided data which did not 


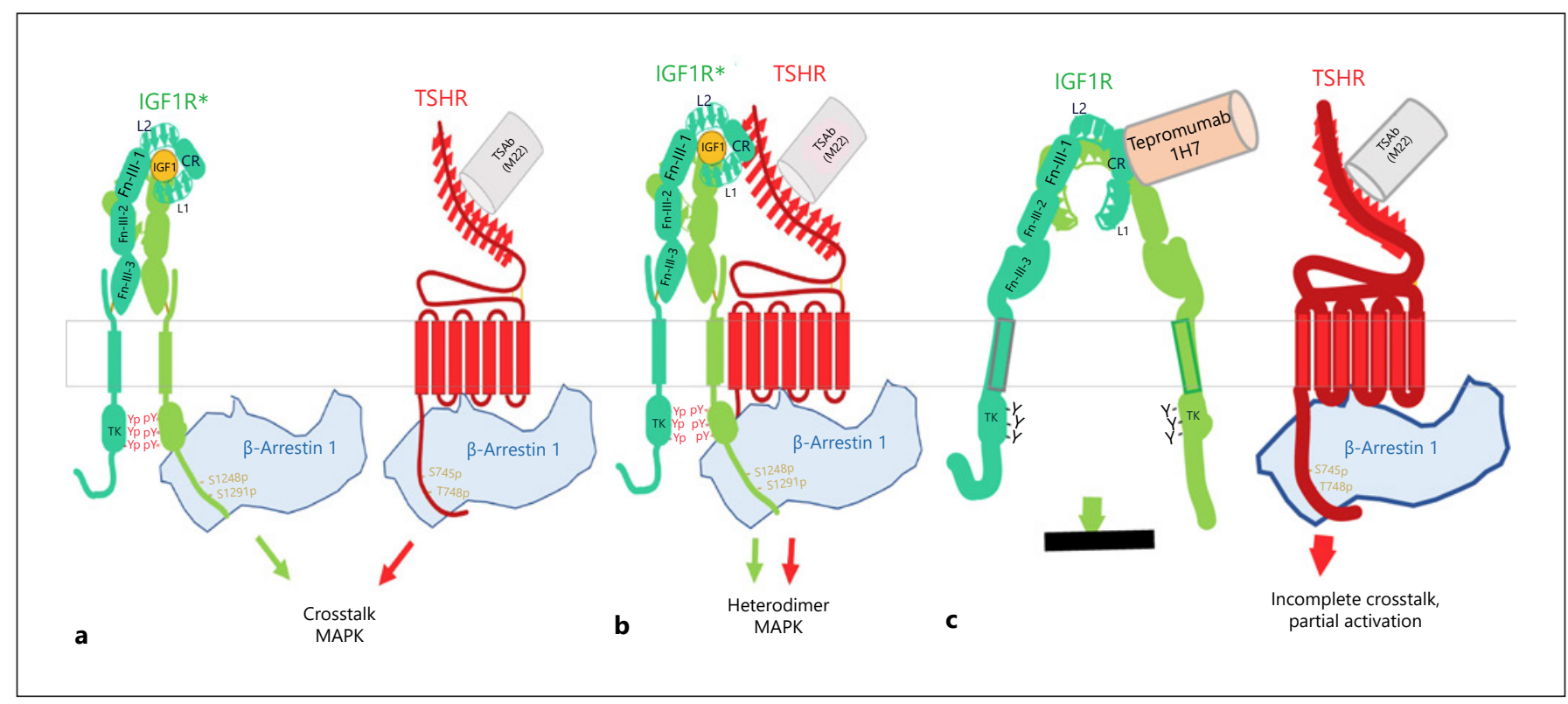

Fig. 3. Schemes of $\beta$-arrestin interactions with IGF-1R and TSHR, which are activated by their respective ligands. Models are based on cryo-EM structure of the IGF-1R [34], $\beta$-arrestin bound GPCR structures [52, 53] and a molecular TSHR structure model [73]. a MAPK activation as a crosstalk via $\beta$-arrestin molecules which bind individually to Ser-phosphorylation sites at C-termini of IGF-1R and TSHR. b MAPK activation via a potential heterodi- mer. $\beta$-Arrestin binds simultaneously to Ser-phosphorylation sites of IGF-1R and the intracellular core of TSHR. c Activation of the IGF-1R is inhibited by the bound antibody teprotumumab (Teprezza) or $1 \mathrm{H} 7$, whereas in case of a crosstalk (common signalosome) partial activation by TSAb-induced TSHR is still possible. Simultaneous inhibition of the IGF-1R and the TSHR would prevent partial activation. support this hypothesis. The authors conclude that GO pathogenesis does not involve directly stimulating IGF$1 \mathrm{R}$ antibodies [48]. Consistent with the latter findings, binding of monoclonal TSAb M22 to IGF-1R could be excluded [49]. Krieger et al. [50] showed that the IGF-1R inhibitory Ig $1 \mathrm{H} 7$ inhibits TSHR/IGF-1R crosstalk-dependent HA synthesis (Fig. 3c), whereas the Ig AF305 did not. Based on the proven proximity between the TSHR and IGF-1R (reviewed in [13]), and the data discussed above, a crosstalk would also be possible in the following scenarios: (1) a signaling overlap of IGF-1R and TSHR is established by interactions of components of the MAPK pathway when $\beta$-arrestin 1 molecules bind to phosphorylated sites of the TSHR (S745p, S748p) [51] and to the Ser-phosphorylations sites S1248p and S1291p [38] of the IGF-1R (Fig. 3a). (2) However, at the TSHR and other GPCRs, $\beta$-arrestin 1 can also bind to the core region at the intracellular side $[52,53]$.

Therefore, from a structural point of view, it is also possible (while not shown experimentally as yet) that one $\beta$-arrestin 1 molecule could simultaneously bind to both the phosphorylated serines of the IGF-1R and the intracellular core domain of TSHR, which would be facilitated by a direct interaction (Fig. 3 b). (3) Finally, a crosstalk might also be possible if agonists, e.g., stimulating antibodies for the TSHR, transactivate also the IGF-1R (Fig. 3b).

Taking the hypothesis above into account, it was not surprising that one recent attempt at targeted therapy in $\mathrm{GO}$ is the blockage of the IGF-1R with antibodies (see next section).

\section{Pharmacological Approaches to Tackle GD and GO}

Here, novel pharmacological approaches and modulators are described mediating direct suppression of pathogenic TSHR activation.

\section{Monoclonal Antibodies}

TSHR-stimulating monoclonal antibodies like M22 [21] bind to the orthosteric binding site of TSH on the extracellular LRRD of the TSHR [32]. It is conceivable that the majority of pathogenic polyclonal TSAb, which activate the TSHR, also bind to this site. TSHR monoclonal antibodies with antagonistic effects like K1-70 [22] 
bind somewhat shifted to the N-terminal end of the extracellular LRR domain and, thus, block the TSHR activation induced by TSH and TSAb (Fig. 1c). Monoclonal TSHR antibodies, such as K1-70 (Table 1), which is already in clinical trials, have a strong pharmacological potential in diseases related to the thyroid [54].

One recent attempt at targeted therapy in GO is the blockage of the IGF-1R with antibodies. Teprotumumab (trade name: Tepezza), an IGF-1R-blocking monoclonal antibody binding to the CR domain of IGF-1R (Fig. 3c; Table 1), blocks its TK activation and induces its internalization [40]. This antibody showed profound treatment effects in 2 phase 3 trials as an immunomodulator to tackle GO, not only in inflammation reduction but also in terms of proptosis reduction $[18,55]$. However, complete remission did still not occur in all of the patients. A partial activation, which originates potentially from residual signaling by the TSHR, still seems to be present. In case of a crosstalk between TSHR and IGF-1R, partial activation of the common signaling pathway by TSAb-induced TSHR could also be possible, although IGF-1R is blocked by an antibody (Fig. 3c). This could hamper complete remission when using teprotumumab. Therefore, simultaneous inhibition of the IGF-1R and TSHR would be a worthwhile strategy to prevent a partial activation of the system. Such a combined blocking of the TSHR and IGF-1R was also suggested in the literature $[46,56]$, especially since it would target both orbitopathy and hyperthyroidism.

\section{TSHR-Derived Peptides of the Extracellular LRRD}

Two types of extracellular LRRD peptides of the TSHR have been reported, either from the concave or convex side of the scythe-leaf-shaped LRRD.

Peptides of the LRRD sequence described by the group of Ungerer [57], such as the TSHR sequence 200-213 (Table 1), showed positive effects on GD and GO manifestations in long-term in vivo mouse GD/GO disease models. Since they are located on the concave side of the LRRD and overlapping with the known interaction site of the monoclonal TSAb M22 [21] (Fig. 1b), it is conceivable that they recognize and bind to TSAb, preventing its interaction with TSHR.

For 2 peptides from the opposite convex side of the LRRD (TSHR sequences 82-95 and 137-150), hyperthyroidism could be reduced in GD patients with combined application (ATX-GD-59; Table 1) in a phase I trial as antigen-specific immunotherapy [58]. The peptides are bound by HLA-DR3 in the antigen-presenting cell and recognized by the T-cell receptor (TCR). These TSHRderived peptides match the HLA-DR3/TCR recognition patterns described and reviewed earlier [59]. It should be mentioned that the peptide FNPCKDIMGY has shown antagonistic effects (Table 1). It corresponds to the sequence of the internal agonist (Fig. 1, 4) but carries the corresponding mutation D409K. It acts not only on TSHR but also on the other glycoproteine hormone receptors, the FSH and LH receptors [28].

\section{Small-Molecule Ligands Blocking TSHR Activation}

Over the last decade, it has been shown that the TSHR and homologous receptors, such as LHR and FSHR, could be modulated at allosteric sites distant from extracellular orthosteric hormone binding sites to produce beneficial effects [60]. Development of small molecules acting on the TSHR as agonists and antagonists have been reviewed previously in 2012 [61], 2015 [19], and 2018 [33]. Unfortunately, blockers for the TSHR are unavailable in the clinics as yet, although GO and GD patients would benefit from such drug development. Such an intervention is especially important for GO patients with muscle fibrosis, which is until now not treatable by any of the available strategies. Muscle fibrosis occurs rather rapidly and is relatively soon irreversible. In in vitro cell cultures using OFs overexpressing the TSHR it could be shown that all signaling pathways leading to HAS production can be blocked by direct inhibition of TSHR signaling, which will probably be the most efficient prevention of muscle fibrosis [62]. The development of small-molecule antagonists of the TSHR, which can be orally administered, is thus of utmost therapeutic interest.

In contrast to the activating autoantibodies, which bind like TSH to the extracellular region of the TSHR (Fig. 1a), small molecules blocking the TSHR as antagonists bind allosterically into a binding pocket located within the heptahelical transmembrane domain (Fig. 1e) $[63,64]$. Drugability of the TSHR by small-molecule antagonists was demonstrated first in 2008 by the weak neutral antagonist, NIDDK/CEB-52 [65], followed in 2010 by the inverse agonist NCGC 00161856 [66]. Derivatives with micromolar activity and the same chemical scaffold were developed by the group of Gershengorn, leading to the neutral antagonist NCGC 00242595 [61] and the inverse agonists NCGC 00229600 (Antag 2) [67] and NCGC 00242364 (Antag 3) [68]. This was also validated in GOinvolved OFs [69]. It should be noted that inverse agonists are not optimal in pharmacological terms to treat disorders accompanied by TSHR hyperstimulation because they also block the physiologically relevant basal constitutive receptor activity, which would consequently necessitate thyroxine application. Moreover, ANTAG2
72

Eur Thyroid J 2020;9(suppl 1):66-77 DOI: $10.1159 / 000511871$
Krause/Eckstein/Schülein 


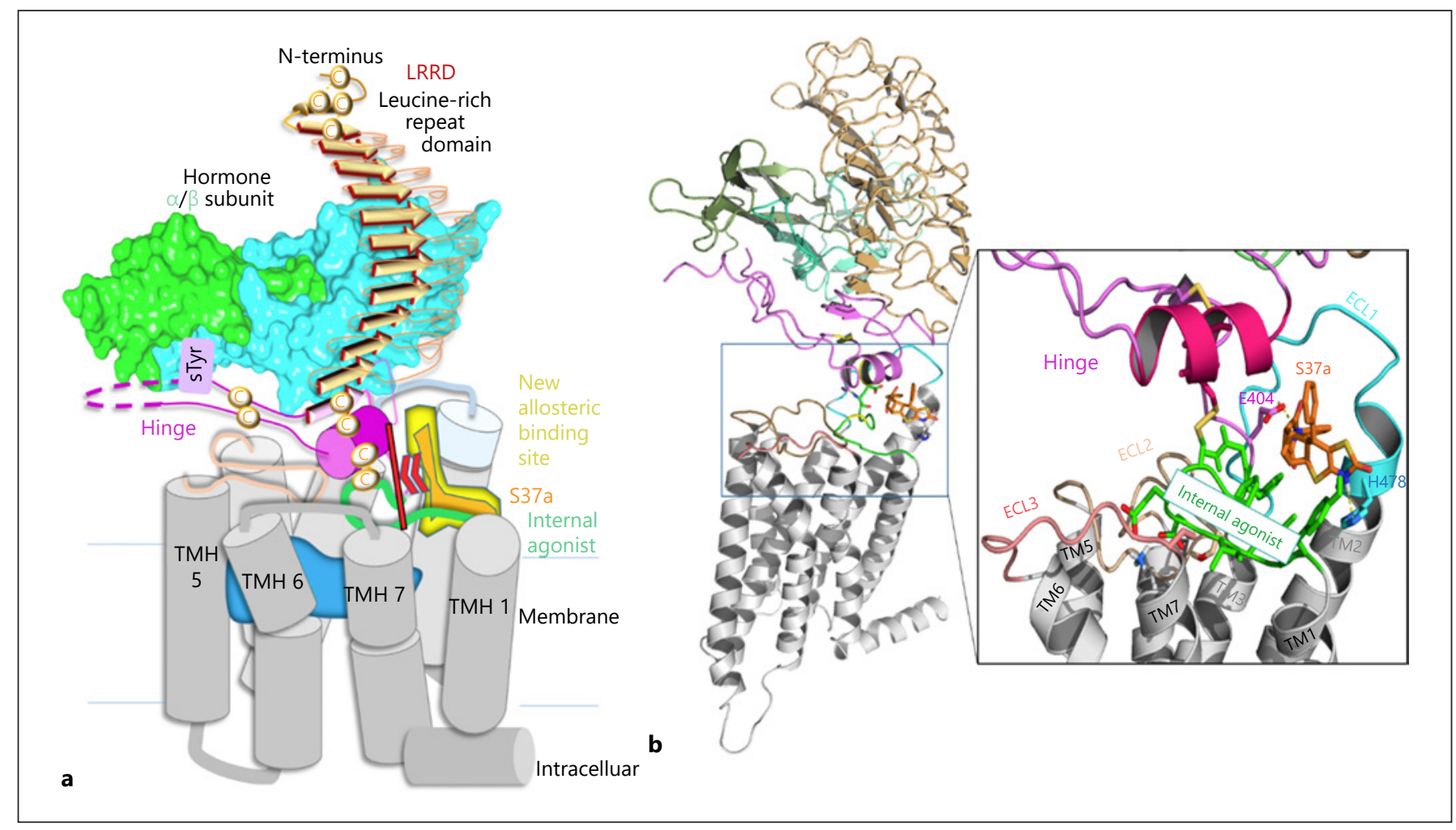

Fig. 4. Antagonist S37a (orange) binds into a newly identified allosteric binding site of TSHR. a Cartoon of the orthosteric binding site of TSH (green/cyan) at the extracellular LRRD (beige). The new allosteric binding site (yellow) is located at the interphase between TMD (grey) and the extracellular domain and is distant from the known allosteric binding site (blue) within the TMD. b Molecular structure model of TSH-bound TSHR. The enlarged inset visualizes details of the S37a interactions between extracellular loop 1 (ECL1, cyan) and the internal agonistic sequence (green) described in [73]. inhibited both IGF-1R-dependent and -independent endpoints at all doses of M22 [50], indicating a crosstalk between TSHR and IGF-1R [49]. Antagonists with different chemical scaffolds were also developed. Van Koppen et al. [70] described a tetrahydroquinoline derivative (Org 274179) and Latif et al. [71] an indole-thiazole derivative with antagonistic activity for the TSHR. The latter was capable of inhibiting TSHR stimulation by GD patient sera and monoclonal-stimulating TSHR antibodies as previously described also for compound VA-K-14 [71]. All of these compounds showed pronounced antagonistic effects on the TSHR. However, they also showed to some extent more or less antagonistic effects at either the homologous FSH or LH receptor.

We also identified by high-throughput screening small molecules with multiple chiral centers as novel TSHR inhibitors. Stereoselective synthesis and enantiomer separation resulted recently in the enantiopure molecule S37a - a micro-molar and, above all, highly TSHR-specif- ic antagonist [72]. S37a forms a bent and rigid structure [73] (Fig. 4b) and inhibits in vitro the TSHR activation by the monoclonal TSAb M22 [23] and the allosteric small molecule agonist compound 2 (C2) [74]. Most importantly, it inhibited TSHR activation by autoimmune TSAb in disease-related ex vivo studies of GO patients' sera [72], too. We narrowed down the binding site of S37a by mutagenesis, homology modeling, and competition studies with C2 and identified a new allosteric inhibition site in the TSHR. It is located between ECL1 and the internal agonist at the interface between TMD and the extracellular domain (Fig. 4). The new allosteric binding site is distant from both the orthosteric TSH binding site and the known allosteric binding site within the TMD [73].

Various compounds (ANTAG3, VA-K-14, and also S37a) with different profiles have been reported to act as preferred allosteric small-molecule inhibitors or negative allosteric modulators of TSHR (Table 1). Of note, all these compounds inhibited cAMP production in the same mi- 


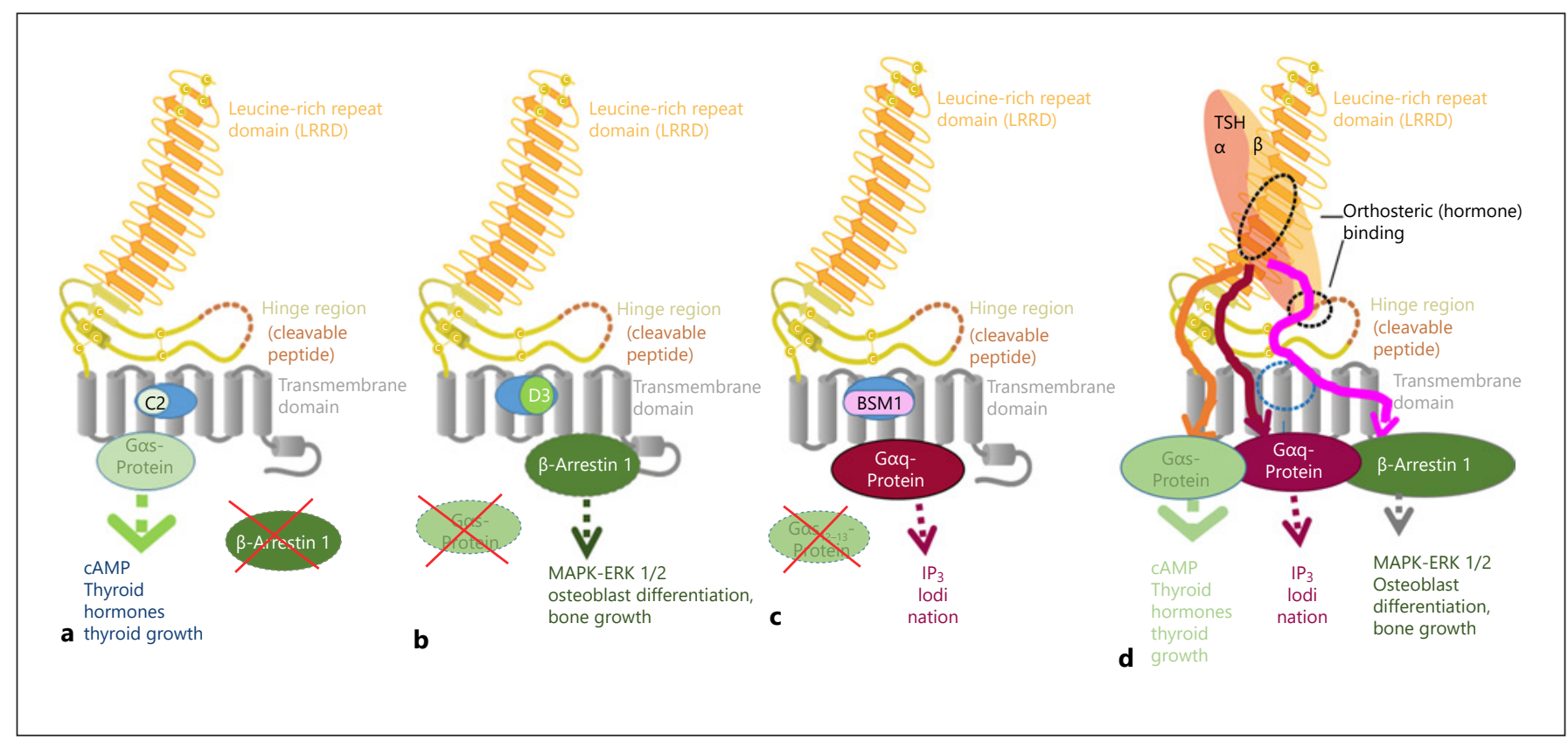

Fig. 5. Biased positive allosteric modulators (agonists) of the TSHR. Schemes of biased interaction in the allosteric binding pocket (blue). a C2 [4] activates only cAMP and not the $\beta$-arrestin pathway. b D3 [77] recruits only $\beta$-arrestin 1 and thereby activates the MAPK-ERK1/2 pathway. Gas is not activated. c BSM1 [78]

cromolar range, meaning that for a reasonable therapeutic application, further effort in potency improvement is needed.

\section{Targeting Biased $\beta$-Arrestin Signaling at the TSHR}

TSH activates at the TSHR mainly $G_{s}$ coupling leading to cAMP formation and thyroid hormone release by thyrocytes. In addition, TSH also stimulates the recruitment of $\beta$-arrestin 1 and 2 to the receptor [29]. At higher TSH concentration, $\mathrm{Gq}[75]$ and $\mathrm{Gi} / \mathrm{o}$ activation [31] were also reported.

Biased ligands can stabilize receptor states that preferentially trigger the interaction with a particular intracellular effector such as G-protein or $\beta$-arrestin.

The small molecule C2 [76] was described as the first biased agonist at the TSHR, activating only the cAMP but not the $\beta$-arrestin pathway [4] (Fig. 5a). The quinazoline derivative D3- $\beta$ Arr was shown to be a biased agonist on TSHR for $\beta$-arrestin (Fig. 5b) and was unable to activate cAMP production [77]. Moreover, a Gq-biased agonist, namely BSM1, was reported recently [78] (Fig. 1c). Biased signaling as described above for IGF-1R thus also occurs at the TSHR. activates only Gaq and neither Gas nor $\mathrm{Ga}_{12-13}$. d Extracellular bound TSH activates all 3 pathways. In conclusion, different intramolecular signaling pathways are present in the TSHR, which could be blocked individually by small molecules.
None of the published small-molecule allosteric antagonist for the TSHR showed a significant biased signaling (reviewed in [33]). The biased agonist data and the fact that TSH activates both G-proteins and $\beta$-arrestin suggest that the different signals in the TSHR are propagated in differentiated intramolecular ways (Fig. 5d). This also opens up the possibility that they could be separately blocked by small-molecule-biased antagonists.

It is conceivable that the different polyclonal autoantibodies in GO patients also induce biased signaling on TSHR. Of note, biased ligands for TSHR are helpful tools to identify specific intramolecular signaling pathways. As an outlook, addressing specific signaling pathways, such as the $\beta$-arrestin pathway, by biased antagonists is also of therapeutic interest, since they could help to avoid unwanted adverse effects.

\section{Conclusions}

Potential GO and GD modulators, which were previously described or are currently under development, are summarized in Table 1. Of note, the IGF-1R blocker te- 
protumumab showed a remarkable effect in 2 phase $3 \mathrm{GO}$ trials, especially in terms of proptosis and inflammation reduction [18]. However, since complete remission did not occur in all of the patients and taking the putative TSHR/IGF-1R cross-interaction into account, additional blocking of the TSAb action at the TSHR might be a promising combined therapeutic approach $[33,69]$. Further investigation of modulators especially addressing their crosstalk/transactivation profile at the TSHR and the IGF-1R are necessary. Moreover, the determinants of the receptors which trigger the different intramolecular signaling pathways must be identified.

Since HLA-DR and TSHR are overexpressed in orbital tissues of GO patients, immunomodulation targeting HLA-DR is also considered as a feasible approach to tackle GO [79], which can be achieved by peptides derived from TSHR [58].

To improve small molecule approaches and the understanding of the multimer interplay of the TSHR and the IGF-1R, we most urgently need to clarify the question whether both receptors indeed form heterodimers or not.

\section{Acknowledgement}

We thank Patrick Marcinkowski for his experimental work on antagonist S37a based on funding provided by the Deutsche Forschungsgemeinschaft (DFG).

\section{Conflict of Interest Statement}

The authors have no conflicts of interest to declare.

\section{Funding Sources}

Grant DFG_KR1273 4/2 to G.K.

\section{Author Contributions}

G.K. designed the concept of the manuscript, created the images, and wrote the manuscript. A.E. and R.S. wrote the manuscript.

\section{References}

1 Endo T, Kobayashi T. Expression of functional TSH receptor in white adipose tissues of hyt/hyt mice induces lipolysis in vivo. Am J Physiol Endocrinol Metab. 2012 Jun; 302(12):E1569-75.

2 Laugwitz KL, Allgeier A, Offermanns S, Spicher K, Van Sande J, Dumont JE, et al. The human thyrotropin receptor: a heptahelical receptor capable of stimulating members of all four $\mathrm{G}$ protein families. Proc Natl Acad Sci USA. 1996 Jan;93(1):116-20.

3 Kero J, Ahmed K, Wettschureck N, Tunaru S, Wintermantel T, Greiner E, et al. Thyrocytespecific Gq/G11 deficiency impairs thyroid function and prevents goiter development. J Clin Invest. 2007 Sep;117(9):2399-407.

4 Boutin A, Eliseeva E, Gershengorn MC, Neumann S. $\beta$-Arrestin-1 mediates thyrotropinenhanced osteoblast differentiation. FASEB J. 2014 Aug;28(8):3446-55.

5 Davies TF, Ando T, Lin RY, Tomer Y, Latif R. Thyrotropin receptor-associated diseases: from adenomata to Graves disease. J Clin Invest. 2005 Aug;115(8):1972-83.

6 Rapoport B, Chazenbalk GD, Jaume JC, McLachlan SM. The thyrotropin (TSH) receptor: interaction with TSH and autoantibodies. Endocr Rev. 1998 Dec;19(6):673-716.

7 Davies TF, Andersen S, Latif R, Nagayama Y, Barbesino G, Brito M, et al. Graves' disease. Nat Rev Dis Primers. 2020 Jul;6(1):52.

8 Wiersinga WM, Bartalena L. Epidemiology and prevention of Graves' ophthalmopathy. Thyroid. 2002 Oct;12(10):855-60.
9 Zhao LQ, Yu DY, Cheng JW. Intravenous glucocorticoids therapy in the treatment of Graves' ophthalmopathy: a systematic review and Meta-analysis. Int J Ophthalmol. 2019 Jul;12(7):1177-86.

10 Salvi M, Vannucchi G, Currò N, Campi I, Covelli D, Dazzi D, et al. Efficacy of B-cell targeted therapy with rituximab in patients with active moderate to severe Graves' orbitopathy: a randomized controlled study. J Clin Endocrinol Metab. 2015 Feb;100(2):422-31.

11 Stan MN, Garrity JA, Carranza Leon BG, Prabin T, Bradley EA, Bahn RS. Randomized controlled trial of rituximab in patients with Graves' orbitopathy. J Clin Endocrinol Metab. 2015 Feb;100(2):432-41.

12 Bahn RS. Current Insights into the Pathogenesis of Graves' Ophthalmopathy. Horm Metab Res. 2015 Sep;47(10):773-8.

13 Krieger CC, Neumann S, Gershengorn MC. TSH/IGF1 receptor crosstalk: mechanism and clinical implications. Pharmacol Ther. 2020 May;209:107502.

14 Eckstein A, Oeverhaus M, Stöhr M, Dekowski D, Berchner-Pfannschmidt U, Esser J, et al. Update endokrine Orbitopathie. Nuklearmediziner. 2019;42(04):291-307.

15 Taylor PN, Zhang L, Lee RW, Muller I, Ezra DG, Dayan CM, et al. New insights into the pathogenesis and nonsurgical management of Graves orbitopathy. Nat Rev Endocrinol. 2020 Feb;16(2):104-16.
16 Smith TJ, Janssen JA. Building the Case for Insulin-Like Growth Factor Receptor-I Involvement in Thyroid-Associated Ophthalmopathy. Front Endocrinol (Lausanne). 2017 Jan;7:167.

17 Smith TJ, Janssen JA. Response to Krieger et al. Re: "TSHR/IGF-1R Cross-Talk, not IGF1R Stimulating Antibodies, Mediates Graves' Ophthalmopathy Pathogenesis" (Thyroid 2017;27:746-747). Thyroid. 2017 Nov;27(11): $1458-9$.

18 Douglas RS, Kahaly GJ, Patel A, Sile S, Thompson EH, Perdok R, et al. Teprotumumab for the Treatment of Active Thyroid Eye Disease. N Engl J Med. 2020 Jan;382(4): 341-52.

19 Davies TF, Latif R. Targeting the thyroidstimulating hormone receptor with small molecule ligands and antibodies. Expert Opin Ther Targets. 2015 Jun;19(6):835-47.

20 Kleinau G, Worth CL, Kreuchwig A, Biebermann H, Marcinkowski P, Scheerer P, et al. Structural-functional features of the thyrotropin receptor: a class A G-Protein-coupled receptor at work. Front Endocrinol (Lausanne). 2017 Apr;8:86.

21 Sanders J, Bolton J, Sanders P, Jeffreys J, Nakatake N, Richards T, et al. Effects of TSH receptor mutations on binding and biological activity of monoclonal antibodies and TSH. Thyroid. 2006 Dec;16(12):1195206 
22 Sanders P, Young S, Sanders J, Kabelis K, Baker S, Sullivan A, et al. Crystal structure of the TSH receptor (TSHR) bound to a blockingtype TSHR autoantibody. J Mol Endocrinol. 2011 Feb;46(2):81-99.

23 Jiang X, Liu H, Chen X, Chen PH, Fischer D, Sriraman V, et al. Structure of follicle-stimulating hormone in complex with the entire ectodomain of its receptor. Proc Natl Acad Sci USA. 2012 Jul;109(31):12491-6.

24 Worth CL, Kleinau G, Krause G. Comparative sequence and structural analyses of Gprotein-coupled receptor crystal structures and implications for molecular models. PLoS One. 2009 Sep;4(9):e7011.

25 Krause G, Kreuchwig A, Kleinau G. Extended and structurally supported insights into extracellular hormone binding, signal transduction and organization of the thyrotropin receptor. PLoS One. 2012;7(12):e52920.

26 Costagliola S, Panneels V, Bonomi M, Koch J, Many MC, Smits G, et al. Tyrosine sulfation is required for agonist recognition by glycoprotein hormone receptors. EMBO J. 2002 Feb;21(4):504-13.

27 Kleinau G, Jäschke H, Neumann S, Lättig J, Paschke R, Krause G. Identification of a novel epitope in the thyroid-stimulating hormone receptor ectodomain acting as intramolecular signaling interface. J Biol Chem. 2004 Dec; 279(49):51590-600.

28 Brüser A, Schulz A, Rothemund S, Ricken A, Calebiro D, Kleinau G, et al. The Activation Mechanism of Glycoprotein Hormone Receptors with Implications in the Cause and Therapy of Endocrine Diseases. J Biol Chem. 2016 Jan;291(2):508-20.

29 Kleinau G, Jaeschke H, Mueller S, Raaka BM, Neumann S, Paschke R, et al. Evidence for cooperative signal triggering at the extracellular loops of the TSH receptor. FASEB J. 2008 Aug;22(8):2798-808.

30 Kleinau G, Jaeschke H, Worth CL, Mueller S, Gonzalez J, Paschke R, et al. Principles and determinants of G-protein coupling by the rhodopsin-like thyrotropin receptor. PLoS One. 2010 Mar;5(3):e9745.

31 Neumann S, Malik SS, Marcus-Samuels B, Eliseeva E, Jang D, Klubo-Gwiezdzinska J, et al. Thyrotropin Causes Dose-dependent Biphasic Regulation of cAMP Production Mediated by Gs and Gi/o Proteins. Mol Pharmacol. 2020 Jan;97(1):2-8.

32 Núñez Miguel R, Sanders J, Chirgadze DY, Furmaniak J, Rees Smith B. Thyroid stimulating autoantibody M22 mimics TSH binding to the TSH receptor leucine rich domain: a comparative structural study of protein-protein interactions. J Mol Endocrinol. 2009 May;42(5):381-95.

33 Krause G, Marcinkowski P. Correction: Intervention Strategies into Glycoprotein Hormone Receptors for Modulating (Mal-)function, with Special Emphasis on the TSH Receptor. Horm Metab Res. 2018 Dec;50(12):e8.
34 Li J, Choi E, Yu H, Bai XC. Structural basis of the activation of type 1 insulin-like growth factor receptor. Nat Commun. 2019 Oct; 10(1):4567.

35 Iacovelli L, Franchetti R, Masini M, De Blasi A. GRK2 and beta-arrestin 1 as negative regulators of thyrotropin receptor-stimulated response. Mol Endocrinol. 1996 Sep;10(9): 1138-46.

36 Lefkowitz RJ, Shenoy SK. Transduction of receptor signals by beta-arrestins. Science. 2005 Apr;308(5721):512-7.

37 Frenzel R, Voigt C, Paschke R. The human thyrotropin receptor is predominantly internalized by $\beta$-arrestin 2 . Endocrinology. 2006 Jun;147(6):3114-22.

38 Girnita L, Worrall C, Takahashi S, Seregard S, Girnita A. Something old, something new and something borrowed: emerging paradigm of insulin-like growth factor type 1 receptor (IGF-1R) signaling regulation. Cell Mol Life Sci. 2014 Jul;71(13):2403-27.

39 Reiter E, Ayoub MA, Pellissier LP, Landomiel F, Musnier A, Tréfier A, et al. $\beta$-arrestin signalling and bias in hormone-responsive GPCRs. Mol Cell Endocrinol. 2017 Jul;449(July): 28-41.

40 Smith TJ, Janssen JA. Insulin-like Growth Factor-I Receptor and Thyroid-Associated Ophthalmopathy. Endocr Rev. 2019 Feb; 40(1):236-67.

41 Zheng H, Shen H, Oprea I, Worrall C, Stefanescu R, Girnita A, et al. $\beta$-Arrestin-biased agonism as the central mechanism of action for insulin-like growth factor 1 receptor-targeting antibodies in Ewing's sarcoma. Proc Natl Acad Sci USA. 2012 Dec;109(50):206205.

42 Girnita L, Girnita A, Larsson O. Mdm2-dependent ubiquitination and degradation of the insulin-like growth factor 1 receptor. Proc Natl Acad Sci USA. 2003 Jul;100(14):824752.

43 Mao Y, Shang Y, Pham VC, Ernst JA, Lill JR, Scales SJ, et al. Polyubiquitination of insulinlike growth factor I receptor (IGF-IR) activation loop promotes antibody-induced receptor internalization and down-regulation. J Biol Chem. 2011 Dec;286(48):41852-61.

44 Tsui S, Naik V, Hoa N, Hwang CJ, Afifiyan NF, Hikim AS, et al. Evidence for an association between thyroid-stimulating hormone and insulin-like growth factor 1 receptors: a tale of rwo antigens implicated in Graves' disease. J Immunol. 2008 Sep;181(6):4397-405.

45 Krieger CC, Boutin A, Jang D, Morgan SJ, Banga JP, Kahaly GJ, et al. Arrestin- $\beta-1$ Physically Scaffolds TSH and IGF1 Receptors to Enable Crosstalk. Endocrinology. 2019 Jun; 160(6):1468-79.

46 Marcus-Samuels B, Krieger CC, Boutin A, Kahaly GJ, Neumann S, Gershengorn MC. Evidence That Graves' Ophthalmopathy Immunoglobulins Do Not Directly Activate IGF-1 Receptors. Thyroid. 2018 May;28(5): $650-5$.
47 Minich WB, Dehina N, Welsink T, Schwiebert C, Morgenthaler NG, Köhrle J, et al. Autoantibodies to the IGF1 receptor in Graves' orbitopathy. J Clin Endocrinol Metab. 2013 Feb; 98(2):752-60.

48 Krieger CC, Neumann S, Gershengorn MC. Is There Evidence for IGF1R-Stimulating Abs in Graves' Orbitopathy Pathogenesis? Int J Mol Sci. 2020 Sep;21(18):E6561.

49 Krieger CC, Neumann S, Marcus-Samuels B, Gershengorn MC. TSHR/IGF-1R Cross-Talk, not IGF-1R Stimulating Antibodies, Mediates Graves' Ophthalmopathy Pathogenesis. Thyroid. 2017 May;27(5):746-7.

50 Krieger CC, Place RF, Bevilacqua C, MarcusSamuels B, Abel BS, Skarulis MC, et al. TSH/ IGF-1 Receptor Cross Talk in Graves' Ophthalmopathy Pathogenesis. J Clin Endocrinol Metab. 2016 Jun;101(6):2340-7.

51 Mayer D, Damberger FF, Samarasimhareddy M, Feldmueller M, Vuckovic Z, Flock T, et al. Distinct $G$ protein-coupled receptor phosphorylation motifs modulate arrestin affinity and activation and global conformation. Nat Commun. 2019 Mar;10(1):1261

52 Latorraca NR, Wang JK, Bauer B, Townshend RJ, Hollingsworth SA, Olivieri JE, et al. Molecular mechanism of GPCR-mediated arrestin activation. Nature. 2018 May;557(7705): 452-6.

53 Lee Y, Warne T, Nehmé R, Pandey S, Dwiwedi-Agnehodi H, Edwards PC, et al. Molecular determinants of $\beta$-arrestin coupling to formoterol-bound $\quad \beta-1 \quad$ adrenoreceptor. BioRxiv. 2020.2 doi. org/10.1101/2020.03.27.011585.

54 Furmaniak J, Sanders J, Núñez Miguel R, Rees Smith B. Mechanisms of Action of TSHR Autoantibodies. Horm Metab Res. 2015 Sep; 47(10):735-52.

55 Smith TJ, Kahaly GJ, Ezra DG, Fleming JC, Dailey RA, Tang RA, et al. Teprotumumab for Thyroid-Associated Ophthalmopathy. N Engl J Med. 2017 May;376(18):1748-61.

56 Krieger CC, Neumann S, Place RF, MarcusSamuels B, Gershengorn MC. Bidirectional TSH and IGF-1 receptor cross talk mediates stimulation of hyaluronan secretion by Graves' disease immunoglobins. J Clin Endocrinol Metab. 2015 Mar;100(3):1071-7.

57 Faßbender J, Holthoff HP, Li Z, Ungerer M. Therapeutic Effects of Short Cyclic and Combined Epitope Peptides in a Long-Term Model of Graves' Disease and Orbitopathy. Thyroid. 2019 Feb;29(2):258-67.

58 Pearce SH, Dayan C, Wraith DC, Barrell K, Olive N, Jansson L, et al. Antigen-Specific Immunotherapy with Thyrotropin Receptor Peptides in Graves' Hyperthyroidism: A Phase I Study. Thyroid. 2019 Jul;29(7):100311.

59 Inaba H, De Groot LJ, Akamizu T. Thyrotropin Receptor Epitope and Human Leukocyte Antigen in Graves' Disease. Front Endocrinol (Lausanne). 2016 Aug;7:120. 
60 Nataraja SG, Yu HN, Palmer SS. Discovery and Development of Small Molecule Allosteric Modulators of Glycoprotein Hormone Receptors. Front Endocrinol (Lausanne). 2015 Sep; $6: 142$.

61 Gershengorn MC, Neumann S. Update in TSH receptor agonists and antagonists. J Clin Endocrinol Metab. 2012 Dec;97(12):4287-92.

62 Zhang L, Grennan-Jones F, Draman MS, Lane C, Morris D, Dayan CM, et al. Possible targets for nonimmunosuppressive therapy of Graves' orbitopathy. J Clin Endocrinol Metab. 2014 Jul;99(7):E1183-90.

63 Haas AK, Kleinau G, Hoyer I, Neumann S, Furkert J, Rutz C, et al. Mutations that silence constitutive signaling activity in the allosteric ligand-binding site of the thyrotropin receptor. Cell Mol Life Sci. 2011 Jan;68(1):159-67.

64 Hoyer I, Haas AK, Kreuchwig A, Schülein R Krause G. Molecular sampling of the allosteric binding pocket of the TSH receptor provides discriminative pharmacophores for antagonist and agonists. Biochem Soc Trans. 2013 Feb;41(1):213-7.

65 Neumann S, Kleinau G, Costanzi S, Moore S Jiang JK, Raaka BM, et al. A low-molecularweight antagonist for the human thyrotropin receptor with therapeutic potential for hyperthyroidism. Endocrinology. 2008 Dec; 149(12):5945-50.

66 Neumann S, Huang W, Eliseeva E, Titus S, Thomas CJ, Gershengorn MC. A small molecule inverse agonist for the human thyroidstimulating hormone receptor. Endocrinology. 2010 Jul;151(7):3454-9.
67 Neumann S, Place RF, Krieger CC, Gershengorn MC. Future Prospects for the Treatment of Graves' Hyperthyroidism and Eye Disease. Horm Metab Res. 2015 Sep;47(10):789-96.

68 Neumann S, Nir EA, Eliseeva E, Huang W, Marugan J, Xiao J, et al. A selective TSH receptor antagonist inhibits stimulation of thyroid function in female mice. Endocrinology. 2014 Jan;155(1):310-4.

69 Neumann S, Pope A, Geras-Raaka E, Raaka BM, Bahn RS, Gershengorn MC. A drug-like antagonist inhibits thyrotropin receptor-mediated stimulation of cAMP production in Graves' orbital fibroblasts. Thyroid. 2012 Aug;22(8):839-43.

70 van Koppen CJ, de Gooyer ME, Karstens WJ, Plate R, Conti PG, van Achterberg TA, et al. Mechanism of action of a nanomolar potent, allosteric antagonist of the thyroid-stimulating hormone receptor. Br J Pharmacol. 2012 Apr;165(7):2314-24.

71 Latif R, Realubit RB, Karan C, Mezei M, Davies TF. TSH Receptor Signaling Abrogation by a Novel Small Molecule. Front Endocrinol (Lausanne). 2016 Sep; 7:130.

72 Marcinkowski P, Hoyer I, Specker E, Furkert J, Rutz C, Neuenschwander M, et al. A new highly thyrotropin receptor-selective smallmolecule antagonist with potential for the treatment of Graves' orbitopathy. Thyroid. 2019 Jan;29(1):111-23

73 Marcinkowski P, Kreuchwig A, Mendieta S, Hoyer I, Witte F, Furkert J, et al. Thyrotropin Receptor: Allosteric Modulators Illuminate Intramolecular Signaling Mechanisms at the Interface of Ecto- and Transmembrane Domain. Mol Pharmacol. 2019 Oct;96(4):45262.
74 Neumann S, Huang W, Titus S, Krause G, Kleinau G, Alberobello AT, et al. Small-molecule agonists for the thyrotropin receptor stimulate thyroid function in human thyrocytes and mice. Proc Natl Acad Sci USA. 2009 Jul;106(30):12471-6.

75 Allen MD, Neumann S, Gershengorn MC. Occupancy of both sites on the thyrotropin (TSH) receptor dimer is necessary for phosphoinositide signaling. FASEB J. 2011;25 (10):3687-94.

76 Titus S, Neumann S, Zheng W, Southall N, Michael S, Klumpp C, et al. Quantitative highthroughput screening using a live-cell cAMP assay identifies small-molecule agonists of the TSH receptor. J Biomol Screen. 2008 Feb; 13(2):120-7.

77 Neumann S, Eliseeva E, Boutin A, Barnaeva E, Ferrer M, Southall N, et al. Discovery of a Positive Allosteric Modulator of the Thyrotropin Receptor: Potentiation of Thyrotropin-Mediated Preosteoblast Differentiation In Vitro. J Pharmacol Exp Ther. 2018 Jan;364(1):38-45.

78 Latif R, Morshed SA, Ma R, Tokat B, Mezei M, Davies TF. A Gq Biased Small Molecule Active at the TSH Receptor. Front Endocrinol (Lausanne). 2020 Jun;11:372.

79 Hai YP, Lee AC, Frommer L, Diana T, Kahaly GJ. Immunohistochemical analysis of human orbital tissue in Graves' orbitopathy. J Endocrinol Invest. $2020 \mathrm{Feb} ; 43(2): 123-37$. 